\title{
Baseband Detection of Bistatic Electron Spin Signals in Magnetic Resonance Force Microscopy
}

\author{
Chun-yu Yip*, Alfred O. Hero*, Daniel Rugar ${ }^{\dagger}$, Jeffrey A. Fessler* \\ * University of Michigan, Ann Arbor, MI \\ ${ }^{\dagger}$ IBM Research Division, Almaden Research Center, San Jose, CA
}

\begin{abstract}
In magnetic resonance force microscopy (MRFM), it is hypothesized that it is possible to detect the presence of a single electron spin in a sample volume by measuring spin-induced attonewton forces using a micromachined cantilever. In the oscillating cantilever driven adiabatic reversals (OSCAR) method for single-spin MRFM, electron spins are manipulated by an external radio-frequency (RF) magnetic field to produce small periodic deviations in the resonant frequency of the cantilever. These deviations can be detected by frequency demodulation followed by a filtered energy detector. In this paper, we present an alternative to energy detection methods, based on optimal detection theory and Gibbs sampling. Receiver operating characteristic (ROC) curves and power curves from simulations are shown for realistic MRFM operating conditions. Surprisingly, the proposed detector performs almost identically to the filtered energy detector for the range of conditions we studied.
\end{abstract}

\section{INTRODUCTION}

Applied physicists recently proposed that magnetic resonance force microscopy (MRFM) can potentially be further extended to the single electron spin level, with sub-angstrom spatial imaging resolution [1-3]. There have been successful experimental demonstrations of detecting micron-size ensembles of electron spins [4] and forces as small as $8 \times 10^{-19}$ Newton [5]. However, detection of an isolated single electron spin has not yet been accomplished. Progress towards this goal will require not only advances in physical measurements, but also a good model of the measurement signal, and a corresponding effective detection algorithm.

In this paper we focus on the oscillating cantilever driven adiabatic reversals (OSCAR) technique [6] in MRFM. OSCAR uses a modulated extemal radio-frequency (RF) magnetic field to manipulate the electron spins in order to produce periodic forces on the oscillating cantilever, which can be detected as small frequency shifts from its natural frequency. Detection of these frequency shifts identifies the presence of electron spins (more details in Section 2). This methodology could potentially be extended to provide single electron spin sensitivity. Unfor-

Research partially supported by DARPA Mosaic program under ARO contract DAAD19-02-C-0055.

Corresponding author: C.Y. Yip (chunyuy@umich.edu) tunately, the spin-induced frequency shift signal becomes extremely weak as the resolution gets close to the single-spin level. Also, the measurements are severely contaminated by thermal noise from various sources. Therefore, signal detection has to operate at extremely low SNRs. Secondly, random spin relaxation leads to random signal parameter changes during measurement.

The baseband amplitude detector and energy detector make the spin presence decisions by thresholding the average absolute amplitude and total energy of the frequency demodulated cantilever position signal, respectively. The baseband filtered energy detector is identical to the energy detector except that it low-pass filters the demodulated signal, according to its decoherence statistics. In this paper, we study a new approach to baseband detection in OSCAR-based MRFM experiments, based on optimal detection theory. The detector is based on a random telegraph model for the baseband measurement signal incorporating Poisson-distributed random spin relaxation times, random initial spin polarity, and additive white Gaussian noise (AWGN). We propose a hybrid detection scheme which combines optimal Bayes and generalized likelihood ratio (GLR) detection principles, implemented with Gibbs sampling. We explore, by simulations, whether the hybrid detector can outperform the detectors listed above, especially the filtered energy detector currently being used in MRFM experiments. In our simulations, the hybrid detector outperformed the amplitude and energy detectors, but surprisingly, performance of the hybrid detector was almost identical to that of the filtered energy detector.

\section{DESCRIPTION OF THE OSCAR EXPERIMENT}

In OSCAR, a submicron ferromagnet is placed at the tip of a cantilever which sits above a sample (Fig. 1). In the presence of an applied RF field, an electron in the sample undergoes magnetic resonance if the RF field frequency matches the Larmor frequency, which is proportional to the strength of field due to the magnetic tip at the electron position. Only those spins that are within a thin resonant slice at a determinate distance will satisfy the condition for magnetic resonance. Oscillation of the magnetic tip leads to oscillation of the tip field strength at the original resonant slice locations. It induces periodic small shifts 
in the Larmor frequency of the spins in that slice. As the RF field frequency is fixed and the Larmor frequency in the slice oscillates, the spins in the slice go through on- and off- resonance states periodically.

Viewing the electron spins as magnetic dipoles, such resonance fluctuation causes the spins to reverse polarity synchronously with the cantilever motion, and such spin loading changes the effective stiffness of the cantilever. Therefore, spins-cantilever interaction can be detected by measuring small shifts in the period of cantilever oscillation using laser interferometric cantilever position sensing. Signal deconvolution of spin ensemble measurements at different locations above the sample can potentially provide single spin resolution [7]. For more details about OSCAR, see [8-10].

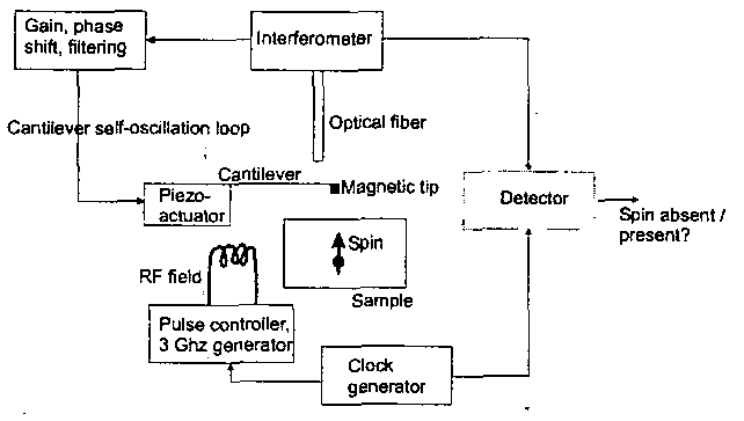

Fig. 1. Schematic of the OSCAR experiment.

A classical mechanical analysis of single-spin-cantilever interaction was proposed by Berman et al. [11] and Rugar et al. [12]. By assumption of linearity, the analysis could be extended to interaction between multiple spins and cantilever.

Let the vertical position of the cantilever tip be denoted by $z$ where $z=0$ denotes its rest position. According to $[11,12]$, under the influence of the external RF field $\mathbf{B}_{1}(t)$, the electronspin force, and random thermal force noise $F_{\mathrm{n}}(t)$, the motion of the cantilever tip can be approximated by the simple harmonic oscillator equation:

$$
m \ddot{z}(t)+\Gamma \dot{\tilde{z}}(t)+(k+\Delta k) z(t)=F_{\mathrm{n}}(t),
$$

where $m$ is the cantilever's effective mass, $k$ is the cantilever spring constant, $\Gamma$ is the friction coefficient characterizing cantilever energy dissipation, and $\Delta k=-\mu G^{2} /\left|B_{1}\right|$ is the shift in spring constant, with $\omega_{o}=\sqrt{k / m}$ being the natural mechanical resonance frequency of the cantilever, $G=\partial B_{0, z} / \partial z$ being the $z$-direction field gradient at the spin location, and $\mu$ being the amplitude of the spin magnetic moment. The spring constant shift results in a shift of $\Delta \omega_{0}$ in the cantilever resonant frequency:

$$
\Delta \omega_{o} \approx-\frac{1}{2} \omega_{o} \frac{\mu G^{2}}{k\left|B_{1}\right|}
$$

In OSCAR, $\mathbf{B}_{1}(t)$ is turned off every $T_{\text {skip }}$ seconds over a half cycle duration $\left(\pi / \omega_{0}\right)$ (Fig. 2$)$ to cause periodic transitions between the spin-lock and anti-spin-lock spin states. Therefore, $\Delta \omega_{o}$ alternates between the two values $\pm \frac{1}{2} \omega_{o}|\mu| G^{2} /\left(k\left|B_{1}\right|\right)$ with period $T_{\text {skip. By setting }} F_{\mathrm{n}}(t)=0$ in (1) and ignoring decay, the solution to (1) can be approximated as the frequency modulated signal:

$$
z(t)=A \cos \left(\omega_{o} t+\int_{0}^{t} \bar{s}\left(t^{\prime}\right) d t^{\prime}+\theta\right) .
$$

Here $A$ is the cantilever oscillation magnitude, $\theta$ is a random phase, and $\bar{s}$ equals a periodic square wave with period $2 T_{\text {skip }}$ and amplitude $\left|\Delta \omega_{o}\right|$ if spin coupling occurs, and 0 otherwise (Fig. 2). Thus spin loading can be detected by frequency demodulation of $z(t)$ to baseband (incorporating subtraction of the known center frequency $\omega_{o}$ ), followed by correlating the baseband signal against the known square wave signal derived from $\mathbf{B}_{1}(t)$, and finally applying a detection algorithm (Fig. 3).

Unfortunately, in a non-ideal experiment, the interferometric cantilever position signal is degraded by thermal noise, which adds a noise floor to the demodulated signal. Another factor is spin relaxation at random time instants during measurement. We assume that spins maintain spin-lock or anti-spin-lock states but spontaneously and asynchronously change polarity during the course of measurement at rate $\lambda$ reversals/second, leading to random transitions of $\Delta \omega_{o}$ between $\pm \frac{1}{2} \omega_{o}|\mu| G^{2} /\left(k\left|B_{1}\right|\right)$. In the following section we develop a detection algorithm using a Poisson random process model for these polarity reversals.
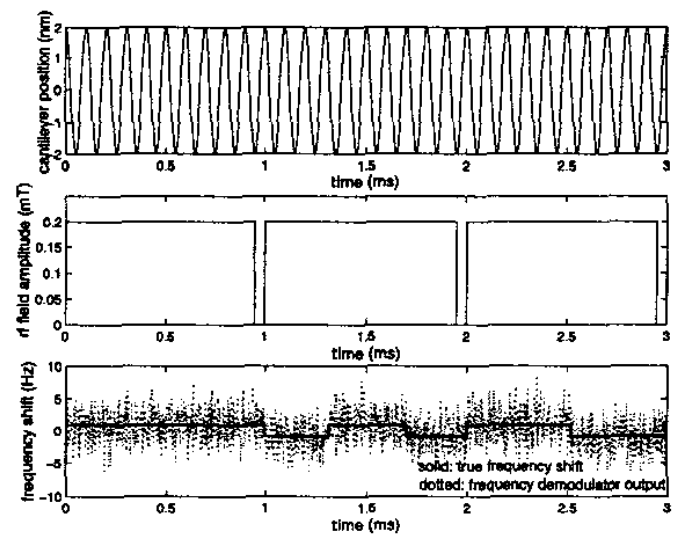

Fig. 2. Top: Sample ideal cantilever position signal from interferometer at $10 \mathrm{kHz}$. Frequency shifts are not detectable by eye. Middle: Amplitude of sample RF magnetic field. $B_{1}(t)$. It has synchronous half-cycle skips at $1 \mathrm{~ms}$, $2 \mathrm{~ms}$, and $3 \mathrm{~ms}$ for creation of spin state transitions. Bottom: Ideal and noisy outputs of the frequency demodulator under the spin presence hypothesis. It has both deterministic transitions due to the RF skips at $1 \mathrm{~ms}, 2 \mathrm{~ms}$ and $3 \mathrm{~ms}$, and random ones due to spin relaxation. The random transitions, $\tau$, occur as a poisson process. The initial polarity is $\phi=1$ for this example. 


\section{SIGNAL MODELING AND DETECTION}

The signal detectors we consider operate on the baseband output signal of a frequency demodulator and a correlator with a square wave reference $p(t) \in\{ \pm 1\}$ of period $2 T_{\text {skip }}^{\text {, whose transitions }}$ are synchronous with the (known) RF turn-off times (Fig. 3).

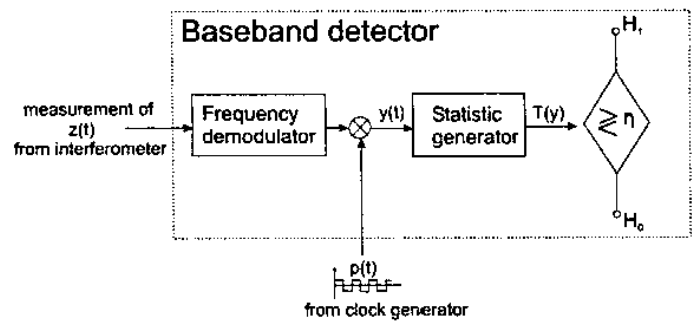

Fig. 3. Baseband detector frequency demodulates the interferometric signal, correlates the output against a square wave $p(t)$ whose transitions are synchronous with the tum-off times of the RF field $\mathbf{B}_{1}(t)$, and generates a test statistic, for detecting presence of a spin.

We model the baseband output $y(t)$ of the frequency demodulator and correlator as a random telegraph signal with AWGN. Let $[0, T]$ be the measurement time period and $\tau=\left\{\tau_{i}\right\}, i=$ $1 . . N$, be the time instants within this period at which random spin reversals occur. We assume $\tau$ are the arrival times of a Poisson process with intensity $\lambda$. Consequently $N$ is a Poisson random variable with rate $\lambda T$ [13]. Thus, $y(t)=s(t)+v(t)$ where $v(t)$ is AWGN with variance $\sigma_{v}^{2}$, and $s(t)$ is a random telegraph signal containing only the random transitions:

$$
s(t)=\phi\left|\Delta \omega_{o}\right| \sum_{i=0}^{N}(-1)^{i} g\left(\frac{t-\tau_{i}}{\tau_{i+1}-\tau_{i}}\right),
$$

where $\phi$ is a random variable that takes on \pm 1 with equal probability, representing a random initial spin polarity, $\tau_{0}=0$, $\tau_{N+1}=T$, and $g(t)$ is the rectangle function: $g(t)=1$ for $t \in(0,1]$ and $g(t)=0$ otherwise. Also, define $s(0)=\phi\left|\Delta \omega_{o}\right|$. If there are no random spin reversals in the time period $[0, T]$, then $s(t)=\phi\left|\Delta \omega_{o}\right|$ is constant over time, which we obtain in (4) by using the convention that when $N=0, \tau_{o}=0$ and $\tau_{1}=T$.

The baseband spin detection problem is to design a test between the two hypotheses:

$$
\begin{array}{cll}
H_{0} \text { (spin absent): } & y(t)=v(t) \\
H_{1} \text { (spin present): } & y(t)=s(t)+v(t)
\end{array}
$$

for $t \in[0, T]$.

\subsection{The Amplitude, Energy, and Filtered Energy Detectors}

One simple detection scheme for the above detection problem is the amplitude detector:

$$
\left|\frac{1}{T} \int_{0}^{T} y\left(t^{\prime}\right) d t^{\prime}\right| \underset{\substack{H_{0} \\ H_{0}}}{\gtrless} \eta
$$

where $\eta$ is a threshold set to satisfy a desired probability of false alarm $\left(P_{F}\right)$ constraint: $P_{\mathrm{F}} \leq \alpha$. In practice, a suitable threshold value can be empirically determined by measurement of the noise variance under the null hypothesis. That applies to the other detectors as well. Other detector options include the energy detector and filtered energy detector:

$$
\int_{0}^{T}\left[\zeta\left(t^{\prime}\right)\right]^{2} d t^{\prime} \stackrel{H_{1}}{\underset{H_{0}}{<}} \eta
$$

where $\eta$ is a threshold chosen to give $P_{\mathrm{F}} \leq \alpha, \zeta(t)=y(t)$ for the energy detector, and $\zeta(t)=y(t) * h(t)$ for the filtered energy detector, with $*$ denoting convolution, and $h(t)$ being the impulse response of a low-pass filter. The bandwidth of the filter should be dependent on the random reversal rate $\lambda$.

\subsection{The Hybrid Bayes/GLR Detector}

For detection of signal with random parameters, the minimum average probability of decision error $\left(\mathrm{min} P_{\mathrm{E}}\right)$ detector is a Bayes likelihood ratio test that averages the omniscient likelihood ratio test statistic over all random parameters [14]:

$$
\begin{aligned}
\log \Lambda(y) & \\
=\log \frac{E_{\boldsymbol{\tau}, N}\left[E_{\dot{\phi}}\left[f\left(y ; \tau, N, \phi \mid H_{\mathbf{1}}\right)\right]\right]}{f\left(y \mid H_{0}\right)} & \underset{H_{0}}{\stackrel{H_{1}}{<}} \eta,
\end{aligned}
$$

where function $f$ is the joint probability density function of $\{y(t)\}_{t \in[0, T]}$ parametrized by the random parameters $\tau, N, \phi$, and $E_{\mathbf{x}}[\cdot A]$ denotes conditional expectation over random variables $\mathbf{x}$ given event $A$. For $\min P_{E}$, threshold $\eta$ should be set to $a \ln \left[P\left(H_{0}\right) / P\left(H_{1}\right)\right]+b$, where $a, b$ are known constants, and $\left(P\left(H_{0}\right), P\left(H_{1}\right)\right)$ are the a priori probabilities of $H_{0}$ and $H_{1}$. However, since the a priori probabilities are unknown, we set $\eta$ to satisfy $P_{\mathrm{F}} \leq \alpha$.

While the expectation over $\phi$ in (8) is simple to evaluate, the expectation over $\{\tau, N\}$ is very difficult since the integration region is of very high (infinite) dimension. An alternative to performing this second expectation is to invoke the GLR principle. The GLR consists of replacing the unknown parameters in (8) by maximum likelihood (ML) estimates:

$$
\begin{aligned}
& \log \Lambda(y) \\
& =\log \frac{\max _{\boldsymbol{\tau}, N}\left\{E_{\phi}\left[f\left(y ; \tau, N, \phi \mid H_{1}\right)\right]\right\}}{f\left(y \mid H_{0}\right)} \underset{H_{0}}{\stackrel{H_{1}}{>}} \eta .
\end{aligned}
$$

where $\eta$ is set to satisfy $P_{\mathrm{F}} \leq \alpha$. As $y(t)$ is a conditionally Gaussian random process given $\tau$ and $N$, the log-likelihood function in ( 9 ) can be simplified by invoking the CameronMartin formula [15]:

$$
\begin{aligned}
\log \Lambda(y) & =\max _{\boldsymbol{\tau}, N}\left\{\log \cosh \left[\frac{1}{\sigma_{v}^{2}} \int_{0}^{T} y(t) s^{+}(t ; \tau, N) d t\right]\right\} \\
& -\frac{1}{2 \sigma_{v}^{2}} \int_{0}^{T}\left(s^{+}(t ; \tau, N)\right)^{2} d t
\end{aligned}
$$


where $s^{+}(t: \tau, N)$ is the synthesized telegraph signal (4) having initial polarity $\phi=1$ (since $E_{\phi}[\cdot]$ is taken) and parametrized by $\tau$ and $N$. In (9) we have averaged over $\phi$ while we have maximized over $\{\boldsymbol{\tau}, N\}$, leading to what we call a hybrid Bayes/GLR test. Notice that the second term in formula (10) is a constant dependent only on SNR, and the $\log \cosh (\cdot)$ function acts as an absolute-value function. Indeed, the hybrid Bayes/GLR detector simply searches for a synthesized sample telegraph signal which has highest absolute correlation with the measurement, and then makes a decision via thresholding the statistic given by the search result.

\subsubsection{Solution via Gibbs Sampling}

The maximization in (10) by exhaustive search over the uncountably infinite-dimensional space of possible parameter values, $\{\tau, N\}$, is impractical. An alternative is to more efficiently search over the space by Gibbs sampling $[16,17]$. Given Poisson intensity $\lambda$, we can generate samples $\left\{\tau^{(j)}, N^{(j)}\right\}$ from the prior Poisson distribution in order to find the maximizer of (10).

The general description of the Gibbs sampler is as follows. Suppose there is a random vector variable $\mathbf{X}=$ $\left[x_{1}, x_{2}, \ldots, x_{p}\right]^{T}$ having density function $f_{\mathbf{X}}$ from which we want to sample. Suppose also that we can simulate the $i$-th element of $\mathbf{X}$ given samples (already simulated) of the other elements:

$$
\begin{aligned}
& X_{i} \mid x_{1}, x_{2}, \ldots, x_{i-1}, x_{i+1}, \ldots, x_{p} \\
\sim & f_{i}\left(x_{i} \mid x_{1}, x_{2}, \ldots, x_{i-1}, x_{i+1}, \ldots, x_{p}\right) \quad \text { for } i=1 . . p
\end{aligned}
$$

Then a Markov sequence, $\mathbf{x}^{(j)}=\left[x_{1}^{(j)}, \ldots, x_{p}^{(j)}\right]^{T}$, can be simulated by the recursion

$$
\begin{aligned}
X_{1}^{(j+1)} & \sim f_{1}\left(x_{1} \mid x_{2}^{(j)}, \ldots, x_{p}^{(j)}\right), \\
X_{2}^{(j+1)} & \sim f_{2}\left(x_{2} \mid x_{1}^{(j+1)}, x_{3}^{(j)}, \ldots, x_{p}^{(j)}\right), \\
\vdots & : \\
X_{p}^{(j+1)} & \sim f_{p}\left(x_{p} \mid x_{1}^{(j+1)}, x_{2}^{(j+1)}, \ldots, x_{p-1}^{(j+1)}\right) .
\end{aligned}
$$

The distribution of $\mathbf{x}^{(j)}$ will converge to $f_{\mathbf{X}}$ after a certain amount of bum-in time [16]. Since the arrival times are distributed as a Poisson process, the univariate conditional distributions (12) are easy to sample from because they are conditionally uniform.

\section{SIMULATION METHODS AND RESULTS}

All of our detection algorithm evaluation were based on simulated receiver operating characteristic (ROC) curves, which were obtained by empirically generating the pairs $\left(P_{\mathrm{F}}, P_{\mathrm{D}}\right)$ for each detector. All simulations was performed in the Matlab 6.5 environment based on the Monte Carlo methodology [16].
For the simulation of one ROC curve point, we generated samples $\left\{y_{d}^{(j)}(n)\right\}, y_{d}(n)=y\left(n T_{s}\right)$, under both Hypothesis 0 and 1 , where $T_{s}$ was the sampling period. The samples were input to the detector being evaluated, and $P_{\mathrm{D}}$ and $P_{F}$ were statistically calculated. 500 detection trials were performed under each hypothesis. For each ROC curve, the above process was repeated with a range of decision threshold values $\eta$. This range was heuristically chosen to adequately sample the domain $P_{\mathrm{F}} \in[0,1]$.

The signal duration $T$ was 3 seconds and the sampling period $T_{s}$ was 0.5 milliseconds. The signal amplitude was fixed at 1 , and the variance of the detector noise (AWGN) was adjusted to give a particular value of SNR, defined as $10 \log _{10}\left[\left(1 /\left(T \sigma_{v}^{2}\right)\right) \int_{0}^{T}|s(t)|^{2} d t\right]$. In all simulations we used 5,000 Gibbs samples for the hybrid Bayes/GLR detector, and the following single-pole low-pass filter for the filtered energy detector:

$$
H(z)=\frac{c\left(1+z^{-1}\right)}{1-a z^{-1}}
$$

where $c=(1-a) / 2, a=(1-\sin (\omega)) / \cos (\omega)$, and $\omega=$ $2 \pi \lambda T_{s}$.

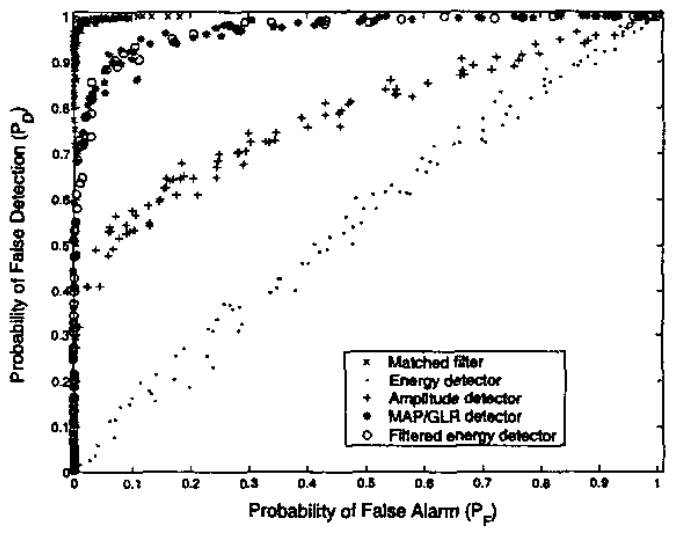

Fig. 4. Simulated ROC curves for the matched filter, energy detector, amplitude detector, filtered energy detector, and hybrid Bayes/GLR detector, at SNR $=-25$ $\mathrm{dB}$ and $\lambda=1$ per-second

The objective of our simulations was to compare the detection performance of the unimplementable, optimal omniscient matched filter (with knowledge of all the parameter values), amplitude detector, energy detector, filtered energy detector, and the proposed hybrid Bayes/GLR detector. ROC curves for SNR $=-25 \mathrm{~dB}$, with $\lambda=1$ per-second, are shown in Fig. 4. Our hybrid Bayes/GLR detector outperformed the amplitude and energy detectors, and was outperformed by the unimplementable matched filter as expected. Surprisingly, it had almost identical performance as the filtered energy detector.

To study how detection performance depends on SNR, we did 
ROC simulations for a range of SNR values with $\lambda=1$ persecond. We did least-square data fitting on ROC data points in the neighborhood of $P_{\mathrm{F}}=0.1$ to find the $P_{\mathrm{D}}$ values corresponding to $P_{\mathrm{F}}=0.1$. Power curves for all detectors as a function of SNR are shown in Fig. 5. We realized that the hybrid detector and filtered energy detector performed almost identically at all the SNR values we investigated. They outperformed the amplitude and energy detectors by almost the same margin: to attain detection performance at $P_{\mathrm{D}}=0.8$, say, the energy detector and amplitude detector required SNRs of at least $-14 \mathrm{~dB}$ and $-17.5 \mathrm{~dB}$, respectively, while the hybrid Bayes/GLR detector and filtered energy detector only required $-26 \mathrm{~dB}$. As compared to the amplitude detector, this represented an improvement of almost $9 \mathrm{~dB}$ in SNR performance. Furthermore, both the hybrid Bayes/GLR and filtered energy detectors were only $4 \mathrm{~dB}$ worse than the performance bound of $-30 \mathrm{~dB}$ established by the matched filter for this level of $P_{\mathrm{F}}$ and $P_{\mathrm{D}}$.

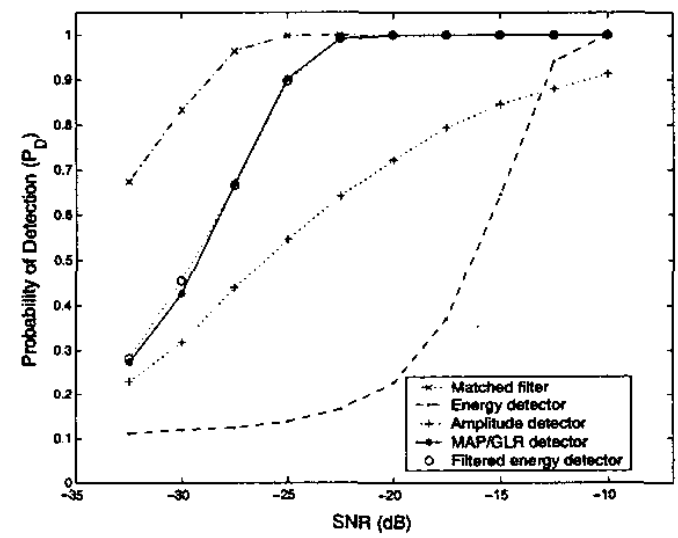

Fig. 5. Power curves ( $P_{\mathrm{D}}$ vs. $\mathrm{SNR}$ ) for the five detectors considered in this paper for $P_{\mathrm{F}}=0.1$ and $\lambda=1$ per-second. At $P_{\mathrm{D}}=0.8$ both the hybrid Bayes/GLR and filtered energy detector performed within $4 \mathrm{~dB}$ of the bound established by the matched filter.

\section{CONCLUSION}

In this paper we described a simple single-spin signal model that physicists have postulated for the OSCAR experiment, and a corresponding hybrid Bayes/GLR approach to detecting the presence of single spin. Assumptions in the model derivation, and even the classical mechanical framework of the single-spincantilever interaction, need to be validated. Nevertheless, it is an important first step to aid MRFM physicists in improving MRFM resolution, and to stimulate more sophisficated physical analysis, signal modeling and detector design.

As expected, with increased computational time, the hybrid Bayes/GLR detector performed significantly better than the amplitude and energy detectors in our simulations, but we were surprised to see that it performed almost identically as the filtered energy detector along the entire power curve. Indeed, it may not be a coincidence. Optimality of the hybrid detector and filtered energy detector are currently under investigation.

While applied physicists are acquiring insights about MRFM theories and experiments, more sophisticated signal models of the cantilever measurements are being postulated, and research in the development of associated detection schemes continues.

\section{REFERENCES}

[1] J. A. Sidles. Nondestructive detection of single-proton magnetic resonance. Appl. Phys. Lett., 58:2854-2856, 1991.

[2] J. A. Sidles, J. L. Garbini, and G. P. Drobny. The theory of oscillatorcoupled magnetic resonance with potential applications to molecular imaging. Rev. Sci. Instrum., 63:3881-3899, 1992.

[3] D. Rugar, B. C. Stipe, H. J. Mamin, C. S. Yannoni, T. D. Stowe, K. Y Yasumura, and T. W. Kenny. Adventures in attonewton force detection. Appl. Phys. A, 72[Suppl.]:S3-S20, 2001.

[4] O. Züger, S. T. Heon, C. S. Yannoni, and D. Rugar. Threedimensional imaging with a nuclear magnetic resonance force microscope. J. Appl. Phys., 79(4):1881-1884, 1996.

[5] H. J. Mamin and D. Rugar. Sub-attonewton force detection at millikelvin temperatures. Appl. Phys. A, 79:3358, 2001.

[6] B. C. Stipe, H. J. Mamin. C. S. Yannoni, T. D. Stowe. T. W. Kenny, and D. Rugar. Electron spin relaxation near a micron-size ferromagnet. Phys. Rev, Lett., 8727(27):7602+, 2001

[7] $O$. Züger and Rugar D. First images from a magnetic resonance force microscope. Appl. Phis. Lett., 63(18):2496-2498, 1993.

[8] J. A. Sidies, J. L. Garbini, K. J. Bruland. D. Rugar, O. Züger, S. Hoen, and C. S. Yannoni. Magnetic resonance force microscopy. Rev: of Modern Phis., 67(1):249-265, 1995.

[9] D. Rugar, O. Züger, S. T. Hoen, C. S. Yannoni, H. M. Vieth, and R. Kendrick. Force detection of nuclear magnetic resonance. Science, 264:1560, 1994.

[10] K. Wago, D. Botkin, C. S. Yannoni, and D. Rugar. Force-detected electronspin resonance: Adiabatic inversion, nutation, and spin echo. Phl's. Rev: $B, 57(2): 1108-1114,1998$.

[11] G. P. Berman, D. 1. Kamenev, and V. 1. Tsifrinovich. Stationary cantilever vibrations in the oscillating cantilever-driven adiabatic reversals - magnetic resonance force microscopy technique. Quant. Phys./020313, 2002.

[12] D. Rugar and R. Budakian. Classical dynamics of a spin interacting with a MRFM cantilever. Technical report, IBM, Almaden Research Center, July 112002 .

[13] W. B. Davenport. Probability and Random Process. McGraw-Hil], New York, 1970.

[14] H. L. Van Trees. Detection, Estimation, and Modulation Theory (Part I). Wiley, New York, 1968.

[15] L. M. Garth and H. V. Poor. Detection of non-gaussian signals: A paradigm for modem statistical signal processing. Proc. IEEE, 82(7):106]-1095, 1994.

[16] C. P. Robert and G. Casella. Monte Carlo Statistical Methods. SpringerVerlag, New York, 1999.

[17] P. Brémaud. Markov Chains - Gibbs Fields, Monte Carlo Simulation, and Queues. Springer-Verlag, New York, 1999. 